\title{
Genome-Wide Association Study for Fatty Acid Composition in American Angus Cattle
}

\author{
Muhammad Dawood ${ }^{1,2}{ }^{\oplus}$, Luke Matthew Kramer ${ }^{3}$, Muhammad Imran Shabbir ${ }^{1}$ and James Mark Reecy ${ }^{3, *}$ \\ 1 Department of Biological Sciences, International Islamic University, Islamabad 44000, Pakistan; \\ muhammad.dawood@uvas.edu.pk (M.D.); imran.shabbir@iiu.edu.pk (M.I.S.) \\ 2 Department of Animal Breeding \& Genetics, University of Veterinary and Animal Sciences, \\ Lahore 54000, Pakistan \\ 3 Department of Animal Science, Iowa State University, 2255 Kildee Hall, Ames, IA 50011, USA; \\ lmkramer@iastate.edu \\ * Correspondence: jreecy@iastate.edu
}

check for updates

Citation: Dawood, M.; Kramer, L.M.; Shabbir, M.I.; Reecy, J.M.

Genome-Wide Association Study for

Fatty Acid Composition in American Angus Cattle. Animals 2021, 11, 2424. https://doi.org/10.3390/ani11082424

\section{Received: 1 July 2021}

Accepted: 12 August 2021

Published: 18 August 2021

Publisher's Note: MDPI stays neutral with regard to jurisdictional claims in published maps and institutional affiliations.

Copyright: (C) 2021 by the authors. Licensee MDPI, Basel, Switzerland. This article is an open access article distributed under the terms and conditions of the Creative Commons Attribution (CC BY) license (https:/ / creativecommons.org/licenses/by/ $4.0 /)$.
Simple Summary: Almost everybody depends on livestock for various reasons directly or indirectly. Consequently, improving livestock production means improving human life. Meat plays important role in human life, as it is good source of protein and energy. Meat composition depends on breed's genetics and environmental factors. Fatty acids (FA) play important role in human diet and health. FA add flavor and taste to meat. Fatty acid composition of meat is a complex polygenic trait that is controlled by genetics and environmental factors. Therefore, the objective of the present study was to identify genomic regions associated with FA composition in American Angus. Thirty-six different genomic regions were identified associated with variation in at least one FA. The genomic regions associated with more than one FA and high genetic variance, harbor good candidate genes (e.g., FABP2, FASN, FADS2, FADS3 and SCD). The identified makers could be used to select for altered FA profile and help to increase the understanding of the genetic basis of FA composition. Furthermore, findings from the present study could help to devise effective breeding plans and selection strategies for the improvement of beef FA profile.

Abstract: Livestock is an important commodity playing a major role in the global economy. Red meat plays an important role in human life, as it is a good source of animal protein and energy. The fatty acid content of beef has been shown to impact the eating experience and nutritional value of beef. Therefore, this study aimed to identify genomic regions which can account for genetic variation in meat fatty acid content. Genotypes imputed to the Illumina BovineHD 770K BeadChip were used in this study. Thirty-six 1-Mb genomic regions with a posterior probability of inclusion (PPI) greater than 0.90 were identified to be associated with variation in the content of at least one fatty acid. The genomic regions $(1 \mathrm{Mb})$ which were associated with more than one fatty acid trait with high genetic variance and harbored good candidate genes were on Chromosome (Chr) 6 (fatty acid binding protein 2), Chr 19 (thyroid hormone receptor alpha, fatty acid synthase), Chr 26 (stearoylCoA desaturase), and Chr 29 (thyroid hormone responsive, fatty acid desaturase 2, and fatty acid desaturase 3). Further studies are required to identify the causal variants within the identified genomic regions. Findings from the present study will help to increase understanding of the variation in fatty acid content of beef and help to enhance selection for beef with improved fatty acid composition.

Keywords: fatty acids; beef; GWAS; high density genotyping; Black Angus

\section{Introduction}

Beef has a high nutritional value, and it is a rich source of minerals, vitamins, and protein. The consumer is becoming more concerned about their health and more conscious about the quality of the meat that they consume. Consumers have been told that beef 
consumption is associated with some serious health issues, such as heart diseases and obesity [1]. However, recent findings have shown that the long-standing belief that beef is associated with cardiovascular disease is incorrect [2-7]. Furthermore, fat is a very important constituent of the human daily diet; it provides energy and also contains essential fatty acids, and adds flavor to food [8]. The fatty acids present in animal tissues can be separated into phospholipid and triacylglyceride fractions [9]. Fatty acid composition and fat content of the beef are associated with the taste, flavor, and sensory properties of the meat [10]. It has been reported that fatty acid composition varies across different breeds and feeding regimes [10,11].

The mechanism that control fatty acid composition of meat is a complex process that is regulated by genetics and environmental factors. There have been several studies published that evaluated the extent to which genetics controlled variation in fatty acid composition in Santa Gertrudis, Brahman, Hereford, Nellore, and Black Angus cattle breeds [12-14]. Identification of genomic markers and regions associated with beef fatty acids could be used to select for an improved fatty acid profile and to alter the saturated to mono- and polyunsaturated fatty acid ratios. The objective of the present study was to identify genomic regions associated with fatty acid composition in American Angus cattle.

\section{Materials and Methods}

\subsection{Animal Selection}

The purebred American Angus cattle used in the current study were reared according to standard animal care procedures, approved by the Iowa State University Animal Care and Use Committee. All the research animals were raised on Iowa State University research demonstration farms.

\subsection{Sampling and DNA Isolation}

A total of 2177 American Black Angus calves sired by 134 sires were used in this study. Blood samples were collected from the jugular vein. DNA samples were collected as previously described by Garmyn et al. [15]. DNA was stored at -20 degrees Celsius until further processing.

\subsection{Genotype Data}

Animals were originally genotyped with either the BovineSNP50 BeadChip (Illumina, San Diego, CA, USA) or the BovineHD BeadChip (Illumina, San Diego, CA, USA) by Neogen GeneSeek Operations (Lincoln, NE, USA). Animals genotyped with the BovineSNP50 BeadChip were imputed to the BovineHD BeadChip SNP density using FImpute [16] and SNPipeline package (Hailin Su, https: / / github.com/cbkmephisto/SNPipeline (accessed on 27 February 2017)) by using 820 Angus individuals originally genotyped on the BovineHD BeadChip. These 820 individuals included animals from the ISU herd and external animals. A filter of 0.05 minor allele frequency was applied and all markers with missing information were excluded. After filtering, a total of 199,431 markers were excluded from analysis, leaving a total of 574,662 markers for data analyses. Genome coordinates are relative to the Bovine UMD 3.1 genome assembly.

\subsection{Fatty Acid Profile}

For fatty acid profile analysis, animals were slaughtered at commercial slaughtering facilities. All the slaughtering procedures were carried out by trained personnel. Carcass data collection, tissue sampling, and fatty acid profile analysis were carried out. Fatty acid composition was analyzed as previously reported [17].

For each fatty acid, phenotypic observations were recorded on a fat percentage basis to estimate the marker effect. In this study, 56 different fatty acid traits were included. 


\subsection{Statistical Analysis}

Imputed genotype data were utilized to estimate the SNP effect associated with fatty acid composition. Statistical analysis was performed using the BayesB method for genomic prediction [18]. Data were analyzed via the following model:

$$
\mathrm{y}=\mathrm{Xb}+\mathrm{Zu}+\mathrm{e}
$$

where $\mathrm{y}$ is the observable value for fatty acid, and $\mathrm{X}$ and $\mathrm{Z}$ are fixed and random effects, respectively. In this model, $b$ is the fixed effect (age, sex, and population mean), $u$ is the random effect marker, and e is the residual effect [18,19]. Fixed effect and covariates included: contemporary group, sex and hot carcass weight, longissimus muscle area at 12th rib, subcutaneous fat thickness at 12 th rib and chemically extracted fat. All the analyses were performed using GenSel software [20].

A chain of 50,000 iterations with the first 5000 as burn-in was used, and the parameter pi $(\pi)$ was set at 0.99906 (99.9\%; approximately 540 SNP markers with a non-zero effect), while genetic and residual variances for each trait were estimated using BayesC (initial variances set as half the total phenotypic variance) before being used in BayesB [21,22]. The posterior probability of inclusion and correlation between QTL and trait were calculated, as described by [20].

\section{Results and Discussion}

\subsection{Fatty Acid Data Statistics}

Summary statistics for the studied fatty acids traits are given in Table 1.

Table 1. Fatty acids and fatty acids groups statistics summary (mean, standard deviation, and coefficient of variance) for all the studied traits.

\begin{tabular}{|c|c|c|c|}
\hline Trait & Mean & SD & $\mathrm{CV} \%$ \\
\hline C10:0 & 0.04 & 0.07 & 196.81 \\
\hline C12:0 & 0.06 & 0.06 & 90.09 \\
\hline C13:0 & 0.01 & 0.07 & 1105.43 \\
\hline C14:0 & 2.71 & 0.58 & 21.21 \\
\hline C14:1 & 0.57 & 0.20 & 34.78 \\
\hline C15:0 & 0.59 & 0.33 & 55.61 \\
\hline C16:0 & 26.57 & 1.80 & 6.79 \\
\hline C16:1 & 3.49 & 0.71 & 20.38 \\
\hline C17:0 & 1.34 & 0.39 & 29.14 \\
\hline C17:1 & 1.07 & 0.37 & 34.65 \\
\hline C18:0 & 13.62 & 1.91 & 14.01 \\
\hline C18:1 c9 & 38.55 & 2.79 & 7.24 \\
\hline C18:1 c11 & 0.10 & 0.10 & 106.32 \\
\hline C18:1 c12 & 0.25 & 0.16 & 63.85 \\
\hline C18:1 c13 & 0.10 & 0.10 & 105.96 \\
\hline C18:1 t6/9 & 0.13 & 0.23 & 178.88 \\
\hline $\mathrm{C} 18: 1 \mathrm{t} 10 / 11$ & 3.58 & 1.39 & 38.84 \\
\hline $\mathrm{C} 18: 1 \mathrm{t} 12$ & 0.07 & 0.24 & 355.96 \\
\hline C18:1 t15 & 1.03 & 0.51 & 48.92 \\
\hline C18:2 & 3.94 & 1.31 & 33.33 \\
\hline
\end{tabular}


Table 1. Cont.

\begin{tabular}{|c|c|c|c|}
\hline Trait & Mean & SD & $\mathrm{CV} \%$ \\
\hline CLA c9t11 & 0.13 & 0.13 & 104.68 \\
\hline CLA t10c12 & 0.05 & 0.09 & 174.15 \\
\hline $\mathrm{C} 18: 3 \mathrm{n} 3$ & 0.17 & 0.16 & 92.47 \\
\hline C18:3 n6 & 0.02 & 0.03 & 222.57 \\
\hline C20:0 & 0.02 & 0.04 & 178.69 \\
\hline C20:1 & 0.09 & 0.11 & 116.14 \\
\hline C20:2 & 0.04 & 0.05 & 131.67 \\
\hline C20:3 n3 & 0.02 & 0.09 & 378.10 \\
\hline C20:3 n6 & 0.12 & 0.17 & 139.19 \\
\hline C20:4 & 0.77 & 0.38 & 48.84 \\
\hline C20:5 & 0.13 & 0.29 & 215.61 \\
\hline C22:0 & 0.11 & 0.15 & 135.98 \\
\hline C22:1 & 0.01 & 0.06 & 1073.49 \\
\hline C22:4 & 0.06 & 0.14 & 215.40 \\
\hline C22:5 & 0.13 & 0.17 & 127.20 \\
\hline C22:6 & 0.08 & 0.16 & 194.83 \\
\hline C23:0 & 0.07 & 0.18 & 256.19 \\
\hline C24:0 & 0.14 & 0.37 & 258.53 \\
\hline SFA & 45.29 & 2.39 & 5.27 \\
\hline MUFA & 49.04 & 2.79 & 5.70 \\
\hline PUFA & 5.67 & 1.85 & 32.66 \\
\hline MCFA & 3.98 & 0.80 & 20.02 \\
\hline LCFA & 96.02 & 0.80 & 0.83 \\
\hline n3 & 0.54 & 0.55 & 101.90 \\
\hline n6 & 5.13 & 1.64 & 32.04 \\
\hline $\mathrm{n} 3 / \mathrm{n} 6$ & 0.11 & 0.13 & 117.97 \\
\hline AI & 0.69 & 0.09 & 12.91 \\
\hline PUFA/SFA & 0.13 & 0.04 & 34.10 \\
\hline UFA/SFA & 1.21 & 0.12 & 9.72 \\
\hline MUFA/SFA & 1.09 & 0.11 & 10.35 \\
\hline C14:1/C14:0 & 0.21 & 0.05 & 25.99 \\
\hline $\mathrm{C} 16: 0 / \mathrm{C} 14: 0$ & 10.19 & 2.05 & 20.07 \\
\hline $\mathrm{C} 16: 1 / \mathrm{C} 16: 0$ & 0.13 & 0.02 & 18.66 \\
\hline C17:1/C17:0 & 0.88 & 1.50 & 170.85 \\
\hline C18:0/C16:0 & 0.52 & 0.09 & 16.80 \\
\hline C16:1-C18:1/C16:0-C18:0 & 1.18 & 0.12 & 10.28 \\
\hline
\end{tabular}

\subsection{Posterior Residual and Genetic Variance, Heritability Estimation}

The SNP-based heritability estimates for fatty acid traits in this study ranged from 0.005 to 0.610 (Table 2). The lower heritability estimates indicated that SNP markers would be poor predictors of fatty acid composition, whereas the higher heritability estimates demonstrate that SNP markers could provide reliable predictions for the content of some 
fatty acids. The highest heritability estimate was 0.610 for C13:0 and 0.478 for C18:1 trans-12. There were ten fatty acid traits that appeared to have moderate heritability values. These ten fatty acids and their respective $\mathrm{h}^{2}$ values were C16:0 (0.20), LCFA (0.231), MCFA (0.232), C14:1 (0.232), n3/n6 (0.237), C14:0 (0.262), C16:1/C16:0 (0.266) C16:0/C14:0 (0.286), C20:0 (0.298), and UFA/SFA (0.359). Heritability estimates for all fatty acid traits are reported in Table 2.

Table 2. Posterior residual variance $\left(\sigma^{2}, \mathrm{~g} \times 10^{-10}\right)$ estimate, genetic variance $\left(\sigma^{2} \mathrm{~g}, \mathrm{~g} \times 10^{-10}\right)$ estimate and the estimated heritability $\left(\mathrm{h}^{2}\right)$ for fatty acids (Fat $\%$ basis).

\begin{tabular}{|c|c|c|c|}
\hline Trait & $\sigma_{e}^{2}, g \times 10^{-10}$ & $\sigma^{2} g, g \times 10^{-10}$ & $h^{2}$ \\
\hline SFA & 3.995 & 1.029 & 0.200 \\
\hline C10:0 & 0.005 & 0.000 & 0.072 \\
\hline C12:0 & 0.003 & 0.000 & 0.021 \\
\hline C13:0 & 0.002 & 0.003 & 0.610 \\
\hline C14:0 & 0.226 & 0.080 & 0.262 \\
\hline C15:0 & 0.071 & 0.002 & 0.023 \\
\hline C16:0 & 2.267 & 0.568 & 0.200 \\
\hline C17:0 & 0.066 & 0.005 & 0.077 \\
\hline C18:0 & 2.133 & 0.426 & 0.166 \\
\hline C20:0 & 0.001 & 0.000 & 0.298 \\
\hline C22:0 & 0.012 & 0.000 & 0.023 \\
\hline C23:0 & 0.031 & 0.001 & 0.019 \\
\hline C24:0 & 0.099 & 0.012 & 0.111 \\
\hline MUFA & 4.932 & 0.879 & 0.151 \\
\hline C14:01 & 0.023 & 0.007 & 0.232 \\
\hline C16:1 & 0.420 & 0.083 & 0.165 \\
\hline C17:1 & 0.058 & 0.003 & 0.045 \\
\hline C18:1 cis-9 & 5.390 & 1.089 & 0.168 \\
\hline C18:1 cis-11 & 0.011 & 0.000 & 0.016 \\
\hline C18:1 cis-12 & 0.018 & 0.002 & 0.104 \\
\hline C18:1 cis-13 & 0.012 & 0.000 & 0.015 \\
\hline C18:1 trans-6/9 & 0.044 & 0.006 & 0.121 \\
\hline C18:1 trans-10/11 & 1.397 & 0.103 & 0.068 \\
\hline C18:1 trans-12 & 0.032 & 0.029 & 0.478 \\
\hline C18:1 trans-15 & 0.217 & 0.006 & 0.029 \\
\hline C20:1 & 0.004 & 0.000 & 0.014 \\
\hline C22:1 & 0.004 & 0.000 & 0.102 \\
\hline PUFA & 2.404 & 0.067 & 0.027 \\
\hline C18:02 & 1.228 & 0.043 & 0.034 \\
\hline C18:3 n-3 & 0.014 & 0.000 & 0.019 \\
\hline C18:3 n-6 & 0.001 & 0.000 & 0.016 \\
\hline
\end{tabular}


Table 2. Cont.

\begin{tabular}{|c|c|c|c|}
\hline Trait & $\sigma_{e}^{2}, g \times 10^{-10}$ & $\sigma_{g}^{2}, g \times 10^{-10}$ & $h^{2}$ \\
\hline C20:2 & 0.002 & 0.000 & 0.016 \\
\hline C20:3 n-3 & 0.009 & 0.000 & 0.014 \\
\hline C20:3 n-6 & 0.025 & 0.002 & 0.062 \\
\hline C20:4 & 0.109 & 0.003 & 0.025 \\
\hline C20:5 & 0.070 & 0.008 & 0.105 \\
\hline $\mathrm{C} 22: 4$ & 0.015 & 0.001 & 0.045 \\
\hline C22:5 & 0.017 & 0.000 & 0.018 \\
\hline C22:6 & 0.019 & 0.004 & 0.191 \\
\hline CLA c9t11 & 0.015 & 0.000 & 0.025 \\
\hline CLA t10c12 & 0.007 & 0.000 & 0.042 \\
\hline$n-3$ & 0.190 & 0.027 & 0.124 \\
\hline$n-6$ & 1.942 & 0.057 & 0.029 \\
\hline$n-3 / n-6$ & 51.527 & 15.985 & 0.237 \\
\hline AI & 11.039 & 1.014 & 0.084 \\
\hline MCFA & 0.444 & 0.134 & 0.232 \\
\hline LCFA & 0.445 & 0.133 & 0.231 \\
\hline MUFA/SFA & 0.012 & 0.002 & 0.134 \\
\hline PUFA/SFA & 3.856 & 0.887 & 0.187 \\
\hline UFA/SFA & 0.007 & 0.004 & 0.359 \\
\hline C14:1/C14:0 & 0.001 & 0.001 & 0.026 \\
\hline C16:0/C14:0 & 2.290 & 0.916 & 0.286 \\
\hline C16:1/C16:0 & 0.000 & 0.000 & 0.266 \\
\hline C16:1,C18:1/C16:0,C18:0 & 0.013 & 0.002 & 0.131 \\
\hline C17:1/C17:0 & 0.058 & 0.003 & 0.045 \\
\hline C18:0/C16:0 & 0.004 & 0.002 & 0.005 \\
\hline
\end{tabular}

All other traits (excluding those described above) appeared to have low heritability values. For these traits, the amount of phenotypic variance explained by the markers was low. The lowest heritability estimated was on fatty acid ratio C18:0/C16:0 (0.005). Previously reported heritability values for fatty acid traits were lower than in the present study. In American Angus, using 50k SNP chip data, the highest heritability value (0.57) was reported for saturated fatty acid C14:0 [18], while in Nellore cattle, the highest reported value was 0.24 for C17:0 and C18:3-n6 [12]. Another study in Canada on beef cattle showed higher $\mathrm{h}^{2}$ values of 0.57 and 0.59 for a saturated fatty acid (C17:0) and monounsaturated fatty acids (C14:1 and C18:1) [23]. A previous study showed that analysis of fatty acid content on a fat percentage basis was able to explain a greater proportion of phenotypic variance by SNP markers, as compared to using fatty acid content on a beef basis [18].

\subsection{Genome-Wide Association Study}

A total of 56 fatty acid traits including saturated, monounsaturated, polyunsaturated, and fatty acid groups were used for genome-wide association studies. The identified genomic regions (1-Mb windows) that showed high genetic variance and posterior probability of inclusion (PPI) greater than $90 \%$ for having non-zero genetic variance or above are presented in Table 3. 
Table 3. 1-Mb SNP windows with PPI $90 \%$ or above for fatty acids on fat percent basis.

\begin{tabular}{|c|c|c|c|c|c|c|}
\hline Trait & PPI ${ }^{1}$ & BTA_Mb & $\begin{array}{c}\text { Start SNP-End } \\
\text { SNP }\end{array}$ & SNP & Var \% & $\begin{array}{c}\text { Map } \\
\text { Position }\end{array}$ \\
\hline C10:0 & 0.944 & 10_99 & $\begin{array}{l}\text { rs381994440- } \\
\text { rs1116598207 }\end{array}$ & 258 & 16.78 & $\begin{array}{l}99005624- \\
99999474\end{array}$ \\
\hline \multirow[t]{4}{*}{ C13:0 } & 0.987 & 21_10 & $\begin{array}{l}\text { rs445662296- } \\
\text { rs722133270 }\end{array}$ & 249 & 7.88 & $\begin{array}{l}10001943- \\
10995552\end{array}$ \\
\hline & 0.989 & 6_65 & $\begin{array}{l}\text { rs723554673- } \\
\text { rs377954800 }\end{array}$ & 159 & 41.63 & $\begin{array}{c}65014180- \\
65999168\end{array}$ \\
\hline & 0.989 & 6_66 & $\begin{array}{l}\text { rs461561099- } \\
\text { rs721295607 }\end{array}$ & 237 & 33.69 & $\begin{array}{c}66005071- \\
66997033\end{array}$ \\
\hline & 0.942 & 6_95 & $\begin{array}{c}\text { rs525139052- } \\
\text { rs714396520 }\end{array}$ & 250 & 3.24 & $\begin{array}{l}95004561- \\
95991513\end{array}$ \\
\hline \multirow[t]{4}{*}{ C14:0 } & 1 & 10_19 & $\begin{array}{l}\text { rs457389817- } \\
\text { rs721999834 }\end{array}$ & 231 & 9.29 & $\begin{array}{l}19000816- \\
19989351\end{array}$ \\
\hline & 1 & 19_51 & $\begin{array}{l}\text { rs475360660- } \\
\text { rs383058850 }\end{array}$ & 204 & 54.85 & $\begin{array}{l}51028723- \\
51996481\end{array}$ \\
\hline & 1 & 26_21 & $\begin{array}{l}\text { rs1118223446- } \\
\text { rs475475724 }\end{array}$ & 186 & 7.39 & $\begin{array}{l}21002029- \\
21996318\end{array}$ \\
\hline & 1 & 29_18 & $\begin{array}{l}\text { rs136831403- } \\
\text { rs438026448 }\end{array}$ & 82 & 23.08 & $\begin{array}{l}18005978- \\
18986358\end{array}$ \\
\hline \multirow[t]{4}{*}{ C14:1 } & 1 & 10_19 & $\begin{array}{c}\text { rs457389817- } \\
\text { rs721999834 }\end{array}$ & 231 & 14.66 & $\begin{array}{l}19000816- \\
19989351\end{array}$ \\
\hline & 1 & $19 \_51$ & $\begin{array}{c}\text { rs475360660- } \\
\text { rs383058850 }\end{array}$ & 204 & 34.52 & $\begin{array}{c}51028723- \\
51996481\end{array}$ \\
\hline & 1 & 26_21 & $\begin{array}{l}\text { rs1118223446- } \\
\text { rs475475724 }\end{array}$ & 186 & 20.19 & $\begin{array}{l}21002029- \\
21996318\end{array}$ \\
\hline & 1 & 29_18 & $\begin{array}{l}\text { rs136831403- } \\
\text { rs438026448 }\end{array}$ & 82 & 18.14 & $\begin{array}{l}18005978- \\
18986358\end{array}$ \\
\hline \multirow[t]{2}{*}{ C16:0 } & 1 & 19_51 & $\begin{array}{l}\text { rs475360660- } \\
\text { rs383058850 }\end{array}$ & 204 & 49.93 & $\begin{array}{l}51028723- \\
51996481\end{array}$ \\
\hline & 1 & 29_18 & $\begin{array}{l}\text { rs136831403- } \\
\text { rs438026448 }\end{array}$ & 82 & 21.78 & $\begin{array}{l}18005978- \\
18986358\end{array}$ \\
\hline \multirow[t]{4}{*}{ C16:1 } & 0.966 & 10_19 & $\begin{array}{l}\text { rs457389817- } \\
\text { rs721999834 }\end{array}$ & 231 & 7.78 & $\begin{array}{l}19000816- \\
19989351\end{array}$ \\
\hline & 1 & $19 \_51$ & $\begin{array}{l}\text { rs475360660- } \\
\text { rs383058850 }\end{array}$ & 204 & 26.23 & $\begin{array}{l}51028723- \\
51996481\end{array}$ \\
\hline & 1 & 26_21 & $\begin{array}{l}\text { rs1118223446- } \\
\text { rs475475724 }\end{array}$ & 186 & 13.85 & $\begin{array}{l}21002029- \\
21996318\end{array}$ \\
\hline & 0.998 & 29_18 & $\begin{array}{l}\text { rs136831403- } \\
\text { rs438026448 }\end{array}$ & 82 & 16.12 & $\begin{array}{l}18005978- \\
18986358\end{array}$ \\
\hline \multirow[t]{2}{*}{ C17:0 } & 0.984 & $19 \_42$ & $\begin{array}{l}\text { rs137786121- } \\
\text { rs466240300 }\end{array}$ & 251 & 22.28 & $\begin{array}{l}42004863- \\
42996553\end{array}$ \\
\hline & 1 & $24 \_49$ & $\begin{array}{l}\text { rs381050710- } \\
\text { rs477123921 }\end{array}$ & 155 & 33.23 & $\begin{array}{l}49001908- \\
49998425\end{array}$ \\
\hline C17:1 & 0.984 & $24 \_49$ & $\begin{array}{l}\text { rs381050710- } \\
\text { rs477123921 }\end{array}$ & 155 & 37.85 & $\begin{array}{l}49001908- \\
49998425\end{array}$ \\
\hline
\end{tabular}


Table 3. Cont.

\begin{tabular}{|c|c|c|c|c|c|c|}
\hline Trait & PPI ${ }^{1}$ & BTA_Mb & $\begin{array}{c}\text { Start SNP-End } \\
\text { SNP }\end{array}$ & SNP & Var $\%$ & $\begin{array}{c}\text { Map } \\
\text { Position }\end{array}$ \\
\hline \multirow[t]{2}{*}{ C18:0 } & 0.92 & 26_21 & $\begin{array}{l}\text { rs1118223446- } \\
\text { rs475475724 }\end{array}$ & 186 & 7.98 & $\begin{array}{l}21002029- \\
21996318\end{array}$ \\
\hline & 1 & 29_18 & $\begin{array}{l}\text { rs136831403- } \\
\text { rs438026448 }\end{array}$ & 82 & 17.1 & $\begin{array}{l}18005978- \\
18986358\end{array}$ \\
\hline \multirow[t]{2}{*}{ C18:1 c9 } & 1 & 19_51 & $\begin{array}{l}\text { rs475360660- } \\
\text { rs383058850 }\end{array}$ & 204 & 50.1 & $\begin{array}{l}51028723- \\
51996481\end{array}$ \\
\hline & 1 & 29_18 & $\begin{array}{l}\text { rs136831403- } \\
\text { rs438026448 }\end{array}$ & 82 & 12.23 & $\begin{array}{l}18005978- \\
18986358\end{array}$ \\
\hline C18:1 c12 & 1 & 26_21 & $\begin{array}{l}\text { rs1118223446- } \\
\text { rs475475724 }\end{array}$ & 186 & 49.36 & $\begin{array}{l}21002029- \\
21996318\end{array}$ \\
\hline $\mathrm{C} 18: 1 \mathrm{t} 6 / 9$ & 0.944 & 27_39 & $\begin{array}{c}\text { rs451168763- } \\
\text { rs381689313 }\end{array}$ & 260 & 8.32 & $\begin{array}{c}39005538- \\
39990529\end{array}$ \\
\hline C18:1 t10/11 & 0.996 & 20_4 & $\begin{array}{l}\text { rs721326040- } \\
\text { rs460617564 }\end{array}$ & 255 & 23.47 & $\begin{array}{l}4010324- \\
4999564\end{array}$ \\
\hline \multirow[t]{7}{*}{$\mathrm{C} 18: 1 \mathrm{t} 12$} & 0.922 & 10_96 & $\begin{array}{c}\text { rs479600948- } \\
\text { rs109335292 }\end{array}$ & 256 & 2.49 & $\begin{array}{l}96019445- \\
96996211\end{array}$ \\
\hline & 1 & 16_46 & $\begin{array}{l}\text { rs478465218- } \\
\text { rs797599032 }\end{array}$ & 224 & 11.46 & $\begin{array}{c}46008112- \\
46996022\end{array}$ \\
\hline & 1 & $16 \_48$ & $\begin{array}{l}\text { rs135228863- } \\
\text { rs474907119 }\end{array}$ & 317 & 9.63 & $\begin{array}{c}48017181- \\
48984950\end{array}$ \\
\hline & 0.98 & 17_65 & $\begin{array}{c}\text { rs378071414- } \\
\text { rs525333053 }\end{array}$ & 285 & 7.51 & $\begin{array}{c}65009493- \\
65999327\end{array}$ \\
\hline & 1 & 21_59 & $\begin{array}{l}\text { rs472316688- } \\
\text { rs451806225 }\end{array}$ & 389 & 2.81 & $\begin{array}{l}59000097- \\
59996736\end{array}$ \\
\hline & 1 & 5_111 & $\begin{array}{l}\text { rs468287514- } \\
\text { rs444667395 }\end{array}$ & 276 & 5.12 & $\begin{array}{l}111007549- \\
111997116\end{array}$ \\
\hline & 0.902 & 5_9 & $\begin{array}{l}\text { rs516462777- } \\
\text { rs1116817234 }\end{array}$ & 152 & 11.55 & $\begin{array}{l}9015235- \\
9989218\end{array}$ \\
\hline C20:3 n6 & 0.924 & 3_86 & $\begin{array}{l}\text { rs718706801- } \\
\text { rs730733704 }\end{array}$ & 264 & 55.3 & $\begin{array}{l}86003522- \\
86991729\end{array}$ \\
\hline \multirow[t]{2}{*}{ C20:5 } & 1 & $29 \_49$ & $\begin{array}{l}\text { rs472519303- } \\
\text { rs526164614 }\end{array}$ & 93 & 22.54 & $\begin{array}{l}49009465- \\
49997333\end{array}$ \\
\hline & 0.991 & 3_86 & $\begin{array}{l}\text { rs718706801- } \\
\text { rs730733704 }\end{array}$ & 264 & 55.49 & $\begin{array}{l}86003522- \\
86991729\end{array}$ \\
\hline \multirow[t]{3}{*}{ C22:1 } & 0.993 & 21_10 & $\begin{array}{l}\text { rs445662296- } \\
\text { rs722133270 }\end{array}$ & 249 & 14.33 & $\begin{array}{l}10001943- \\
10995552\end{array}$ \\
\hline & 0.987 & 30_71 & $\begin{array}{l}\text { rs458478290- } \\
\text { rs481059659 }\end{array}$ & 65 & 36.65 & $\begin{array}{l}71227458- \\
71976081\end{array}$ \\
\hline & 0.987 & 30_72 & $\begin{array}{l}\text { rs524807927- } \\
\text { rs135609351 }\end{array}$ & 29 & 88.09 & $\begin{array}{l}72004959- \\
72982639\end{array}$ \\
\hline C22:4 & 0.924 & 25_11 & $\begin{array}{l}\text { rs467215611- } \\
\text { rs456314684 }\end{array}$ & 387 & 27.95 & $\begin{array}{l}11001504- \\
11999813\end{array}$ \\
\hline \multirow[t]{2}{*}{$\mathrm{C} 22: 6$} & 0.96 & 25_11 & $\begin{array}{l}\text { rs467215611- } \\
\text { rs456314684 }\end{array}$ & 387 & 6.24 & $\begin{array}{l}11001504- \\
11999813\end{array}$ \\
\hline & 1 & 3_86 & $\begin{array}{l}\text { rs718706801- } \\
\text { rs730733704 }\end{array}$ & 264 & 76.04 & $\begin{array}{c}86003522- \\
86991729\end{array}$ \\
\hline
\end{tabular}


Table 3. Cont.

\begin{tabular}{|c|c|c|c|c|c|c|}
\hline Trait & PPI ${ }^{1}$ & BTA_Mb & $\begin{array}{c}\text { Start SNP-End } \\
\text { SNP }\end{array}$ & SNP & Var \% & $\begin{array}{c}\text { Map } \\
\text { Position }\end{array}$ \\
\hline \multirow[t]{2}{*}{ C24:0 } & 1 & $16 \_65$ & $\begin{array}{l}\text { rs468954509- } \\
\text { rs1117996716 }\end{array}$ & 305 & 50.06 & $\begin{array}{l}65001270- \\
65996849\end{array}$ \\
\hline & 0.946 & 3_86 & $\begin{array}{l}\text { rs718706801- } \\
\text { rs730733704 }\end{array}$ & 264 & 39.59 & $\begin{array}{l}86003522- \\
86991729\end{array}$ \\
\hline \multirow[t]{5}{*}{ SFA } & 0.949 & 1_115 & $\begin{array}{c}\text { rs436612027- } \\
\text { rs715205098 }\end{array}$ & 224 & 6.43 & $\begin{array}{l}115000891- \\
115995052\end{array}$ \\
\hline & 0.998 & $16 \_4$ & $\begin{array}{l}\text { rs450830345- } \\
\text { rs379811569 }\end{array}$ & 313 & 6.69 & $\begin{array}{l}4004764- \\
4992166\end{array}$ \\
\hline & 1 & 19_51 & $\begin{array}{c}\text { rs475360660- } \\
\text { rs383058850 }\end{array}$ & 204 & 31.53 & $\begin{array}{c}51028723- \\
51996481\end{array}$ \\
\hline & 1 & 26_21 & $\begin{array}{l}\text { rs1118223446- } \\
\text { rs475475724 }\end{array}$ & 186 & 12.23 & $\begin{array}{l}21002029- \\
21996318\end{array}$ \\
\hline & 0.951 & 7_93 & $\begin{array}{c}\text { rs443092875- } \\
\text { rs378089989 }\end{array}$ & 143 & 6.46 & $\begin{array}{l}93002992- \\
93993941\end{array}$ \\
\hline \multirow[t]{3}{*}{ MUFA } & 0.926 & $16 \_4$ & $\begin{array}{l}\text { rs450830345- } \\
\text { rs379811569 }\end{array}$ & 313 & 5.63 & $\begin{array}{l}4004764- \\
4992166\end{array}$ \\
\hline & 1 & $19 \_51$ & $\begin{array}{c}\text { rs475360660- } \\
\text { rs383058850 }\end{array}$ & 204 & 35.94 & $\begin{array}{c}51028723- \\
51996481\end{array}$ \\
\hline & 0.913 & $26 \_21$ & $\begin{array}{l}\text { rs1118223446- } \\
\text { rs475475724 }\end{array}$ & 186 & 9.54 & $\begin{array}{l}21002029- \\
21996318\end{array}$ \\
\hline \multirow[t]{3}{*}{ MCFA } & 1 & 10_19 & $\begin{array}{l}\text { rs457389817- } \\
\text { rs721999834 }\end{array}$ & 231 & 11.22 & $\begin{array}{l}19000816- \\
19989351\end{array}$ \\
\hline & 1 & $19 \_51$ & $\begin{array}{l}\text { rs475360660- } \\
\text { rs383058850 }\end{array}$ & 204 & 57.4 & $\begin{array}{l}51028723- \\
51996481\end{array}$ \\
\hline & 1 & 29_18 & $\begin{array}{l}\text { rs136831403- } \\
\text { rs438026448 }\end{array}$ & 82 & 23.19 & $\begin{array}{l}18005978- \\
18986358\end{array}$ \\
\hline \multirow[t]{3}{*}{ LCFA } & 1 & 10_19 & $\begin{array}{l}\text { rs457389817- } \\
\text { rs721999834 }\end{array}$ & 231 & 11.35 & $\begin{array}{l}19000816- \\
19989351\end{array}$ \\
\hline & 1 & 19_51 & $\begin{array}{l}\text { rs475360660- } \\
\text { rs383058850 }\end{array}$ & 204 & 57.3 & $\begin{array}{l}51028723- \\
51996481\end{array}$ \\
\hline & 1 & 29_18 & $\begin{array}{l}\text { rs136831403- } \\
\text { rs438026448 }\end{array}$ & 82 & 23.02 & $\begin{array}{l}18005978- \\
18986358\end{array}$ \\
\hline \multirow[t]{2}{*}{ n3 } & 1 & $29 \_49$ & $\begin{array}{l}\text { rs472519303- } \\
\text { rs526164614 }\end{array}$ & 93 & 11.73 & $\begin{array}{l}49009465- \\
49997333\end{array}$ \\
\hline & 1 & 3_86 & $\begin{array}{l}\text { rs718706801- } \\
\text { rs730733704 }\end{array}$ & 264 & 76.51 & $\begin{array}{l}86003522- \\
86991729\end{array}$ \\
\hline \multirow[t]{5}{*}{ UFA/SFA } & 0.982 & 1_115 & $\begin{array}{l}\text { rs436612027- } \\
\text { rs715205098 }\end{array}$ & 224 & 3.92 & $\begin{array}{l}115000891- \\
115995052\end{array}$ \\
\hline & 0.991 & 16_4 & $\begin{array}{l}\text { rs450830345- } \\
\text { rs379811569 }\end{array}$ & 313 & 3.24 & $\begin{array}{l}4004764- \\
4992166\end{array}$ \\
\hline & 1 & 19_51 & $\begin{array}{c}\text { rs475360660- } \\
\text { rs383058850 }\end{array}$ & 204 & 15.35 & $\begin{array}{c}51028723- \\
51996481\end{array}$ \\
\hline & 0.962 & 22_9 & $\begin{array}{l}\text { rs446574361- } \\
\text { rs134422456 }\end{array}$ & 247 & 1.87 & $\begin{array}{l}9004252- \\
9998969\end{array}$ \\
\hline & 0.989 & 26_21 & $\begin{array}{c}\text { rs1118223446- } \\
\text { rs475475724 }\end{array}$ & 186 & 5.34 & $\begin{array}{l}21002029- \\
21996318\end{array}$ \\
\hline
\end{tabular}


Table 3. Cont.

\begin{tabular}{|c|c|c|c|c|c|c|}
\hline Trait & PPI ${ }^{1}$ & BTA_Mb & $\begin{array}{c}\text { Start SNP-End } \\
\text { SNP }\end{array}$ & SNP & Var \% & $\begin{array}{c}\text { Map } \\
\text { Position }\end{array}$ \\
\hline & 0.931 & 26_32 & $\begin{array}{l}\text { rs136160709- } \\
\text { rs382889271 }\end{array}$ & 271 & 2.06 & $\begin{array}{l}32010478- \\
32984426\end{array}$ \\
\hline & 0.993 & 7_93 & $\begin{array}{l}\text { rs443092875- } \\
\text { rs378089989 }\end{array}$ & 143 & 3.86 & $\begin{array}{r}93002992- \\
93993941\end{array}$ \\
\hline \multirow[t]{3}{*}{ MUFA/SFA } & 0.989 & $16 \_4$ & $\begin{array}{l}\text { rs450830345- } \\
\text { rs379811569 }\end{array}$ & 313 & 5.27 & $\begin{array}{l}4004764- \\
4992166\end{array}$ \\
\hline & 1 & $19 \_51$ & $\begin{array}{l}\text { rs475360660- } \\
\text { rs383058850 }\end{array}$ & 204 & 35.99 & $\begin{array}{l}51028723- \\
51996481\end{array}$ \\
\hline & 0.904 & 26_21 & $\begin{array}{l}\text { rs1118223446- } \\
\text { rs475475724 }\end{array}$ & 186 & 7.44 & $\begin{array}{l}21002029 \\
21996318\end{array}$ \\
\hline C14:1/C14:0 & 1 & $26 \_21$ & $\begin{array}{l}\text { rs1118223446- } \\
\text { rs475475724 }\end{array}$ & 186 & 52 & $\begin{array}{l}21002029 \\
21996318\end{array}$ \\
\hline \multirow[t]{4}{*}{ C16:0/C14:0 } & 1 & $19 \_51$ & $\begin{array}{l}\text { rs475360660- } \\
\text { rs383058850 }\end{array}$ & 204 & 34.55 & $\begin{array}{l}51028723- \\
51996481\end{array}$ \\
\hline & 1 & $26 \_21$ & $\begin{array}{l}\text { rs1118223446- } \\
\text { rs475475724 }\end{array}$ & 186 & 6.99 & $\begin{array}{l}21002029 \\
21996318\end{array}$ \\
\hline & 1 & 29_18 & $\begin{array}{l}\text { rs136831403- } \\
\text { rs438026448 }\end{array}$ & 82 & 15.52 & $\begin{array}{l}18005978- \\
18986358\end{array}$ \\
\hline & 0.947 & $29 \_42$ & $\begin{array}{l}\text { rs379690091- } \\
\text { rs463588285 }\end{array}$ & 155 & 5.38 & $\begin{array}{l}42001720 \\
42991376\end{array}$ \\
\hline \multirow[t]{6}{*}{ C16:1/C16:0 } & 0.984 & 10_19 & $\begin{array}{c}\text { rs457389817- } \\
\text { rs721999834 }\end{array}$ & 231 & 5.7 & $\begin{array}{l}19000816- \\
19989351\end{array}$ \\
\hline & 0.989 & $19 \_51$ & $\begin{array}{c}\text { rs475360660- } \\
\text { rs383058850 }\end{array}$ & 204 & 6.14 & $\begin{array}{l}51028723- \\
51996481\end{array}$ \\
\hline & 1 & $26 \_21$ & $\begin{array}{l}\text { rs1118223446- } \\
\text { rs475475724 }\end{array}$ & 186 & 8.4 & $\begin{array}{c}21002029 \\
21996318\end{array}$ \\
\hline & 0.96 & 26_29 & $\begin{array}{l}\text { rs380753352- } \\
\text { rs42912734 }\end{array}$ & 240 & 3.41 & $\begin{array}{l}29000086- \\
29997851\end{array}$ \\
\hline & 0.964 & 29_18 & $\begin{array}{l}\text { rs136831403- } \\
\text { rs438026448 }\end{array}$ & 82 & 4.26 & $\begin{array}{l}18005978- \\
18986358\end{array}$ \\
\hline & 0.935 & $6 \_7$ & $\begin{array}{l}\text { rs43449965- } \\
\text { rs797424312 }\end{array}$ & 218 & 3.36 & $\begin{array}{l}7050818- \\
7999410\end{array}$ \\
\hline \multirow[t]{4}{*}{ C18:0/C16:0 } & 0.824 & $15 \_75$ & $\begin{array}{c}\text { rs474144146- } \\
\text { rs799862808 }\end{array}$ & 288 & 2.87 & $\begin{array}{l}75000095- \\
75998710\end{array}$ \\
\hline & 1 & $19 \_51$ & $\begin{array}{c}\text { rs475360660- } \\
\text { rs383058850 }\end{array}$ & 204 & 13.38 & $\begin{array}{l}51028723- \\
51996481\end{array}$ \\
\hline & 0.9 & 21_22 & $\begin{array}{l}\text { rs449389929- } \\
\text { rs437752920 }\end{array}$ & 258 & 2.43 & $\begin{array}{l}22011632- \\
22997995\end{array}$ \\
\hline & 1 & 29_18 & $\begin{array}{l}\text { rs136831403- } \\
\text { rs438026448 }\end{array}$ & 82 & 15.26 & $\begin{array}{l}18005978- \\
18986358\end{array}$ \\
\hline \multirow[t]{2}{*}{$\begin{array}{l}\text { C16:1,C18:1/ } \\
\text { C16:0,C18:0 }\end{array}$} & 0.995 & 16_4 & $\begin{array}{l}\text { rs450830345- } \\
\text { rs379811569 }\end{array}$ & 313 & 5.62 & $\begin{array}{l}4004764- \\
4992166\end{array}$ \\
\hline & 1 & $19 \_51$ & $\begin{array}{l}\text { rs475360660- } \\
\text { rs383058850 }\end{array}$ & 204 & 31.51 & $\begin{array}{l}51028723- \\
51996481\end{array}$ \\
\hline
\end{tabular}

${ }^{1}$ PPI = Posterior Probability of Inclusion.

The highest estimated genetic variance explained by a single SNP window was $88.09 \%$ for the fatty acid C22:1, while the window with the lowest estimated genetic variance, $1.72 \%$, 
was for fatty acid C18:1t12. Regarding the SNP window which explained the highest level of genetic variance (30_72), there was a potential candidate gene, phosphatidylinositol specific phospholipase C X domain containing 1 (PLCXD1), on the pseudo-autosomal region (PAR). This gene is X-linked in ruminants. PLCXD gene products are phosphodiesterases involved in the regulation of cytosolic calcium and have protein kinase activity [24,25].

This study identified a total of 36 different $1-\mathrm{Mb}$ SNP windows that were associated with fatty acid content of skeletal muscle. Three windows (19_51,26_21, and 29_18) appeared to be associated with most beef fatty acid traits. These three windows were previously reported in the same Black Angus population using the Bovine SNP50 BeadChip [18]. Many of the 1-Mb SNP windows were associated with more than one fatty acid trait. A genomic region on chromosome 19 (at $51 \mathrm{Mb}$ ) was associated with 15 fatty acid traits (LCFA, MCFA, MUFA, MUFA/SFA, SFA, UFA/SFA, C14:0, C14:1, C16:0, C16:1, C18:1c9, C16:0/C14:0, C16:1/C16:0, C16:1-C18:1/C16:0-C18:0, and C18:0/C16:0). This region was also previously reported to be associated with important fatty acid traits $[18,26]$. This SNP window contains a good candidate gene-fatty acid synthase (FASN). This gene has been reported to be involved in beef fatty acid composition [27]. It has also been reported to be associated with adipose composition, milk fatty acid composition, and milk fat content in many different breeds of cattle. These reports indicated that this gene has a pivotal role and is an important candidate gene for fatty acid composition [26,28-31].

Similar to the window at $51 \mathrm{Mb}$ on chromosome 19, there were additional SNP windows that were associated with more than ten fatty acid traits, including a window at $21 \mathrm{Mb}$ on Chromosome 26, which was associated with $13 \mathrm{FA}$ traits, and a window at $18 \mathrm{Mb}$ on Chromosome 29, which was associated with 11 FA traits. These two SNP windows were also previously reported to be associated with various FA traits $[18,26]$. These two regions harbor good candidate genes for fatty acid composition, including stearoyl-CoA desaturase $(S C D)$ and thyroid hormone responsive (THRSP). Previous studies have reported that SCD is associated with meat fat composition and milk fat composition [28-31]. Thyroid hormone responsive and stearoyl-CoA desaturase is involved in fatty acid synthesis [32]. Variants in the THRSP gene have been shown to be associated with the synthesis of beef fatty acids, which are expected to have a direct impact on beef quality $[18,33]$. It has also been reported that both SCD and THRSP genes are involved in lipid metabolism in cattle [34]. The 1-Mb SNP windows that harbor good candidate genes for fatty acid synthesis and fat regulation are shown in Table 4.

In the present GWAS, some new genomic windows were identified which were not previously reported by any study. These new SNP windows are associated with different fatty acid traits and harbor good candidate genes for fatty acid composition. On chromosome 16, a 1-Mb SNP window at $4 \mathrm{Mb}$ was associated with SFA, MUFA, MUFA/SFA, UFA/SFA, and C16:1, C18:1/C16:0, C18:0. This window contains good candidate genes-6-phosphofructo-2-kinase/fructose-2,6-biphosphatase 2 (PFKFB2) and peptidase M20 domain-containing 1 (PM20D1). The PFKFB2 gene has a role in degradation and synthesis of fructose-2,6-biphosphate [26]. It has been previously reported that a QTL spanning this region is related to fat thickness at the 12th rib in American Angus [35]. Peptidase M20 domain-containing 1 (PM20D1) is an enzyme that synthesizes N-acyl amino acids (NAAs). NAAs are bioactive lipids composed of fatty acyl chains. PM20D1 regulates the condensation and hydrolysis of $\mathrm{N}$-acyl amino acids from free amino acids and fatty acids [36-38]. Fatty acid binding protein 2 (FABP2) may have a role in lipogenesis and adipose tissue weight variability [39]. This gene has not been identified in previous GWAS as having a significant association with fatty acid composition [40]. Bardet-Biedl syndrome 4 (BBS4) is involved in the secretion and expression of Follistatin-like 1 (FSTL1), which is associated with adipogenesis. BBS4 also plays a role in fatty acid profile, lipolysis, and fat accumulation [41,42]. Acetyl-CoA acyltransferase 2 (ACAA2) codes for an enzyme from the thiolase family. This enzyme is involved in elongation and degradation of fatty acids. It has been associated with milk yield and fat yield in dairy sheep [43]. Fatty acid desaturase 2 (FADS2) and fatty acid desaturase 3 (FADS3) belong to the fatty acid desaturase 
family. This family of genes creates a cis double bond in FA chains at specific sites and is associated with desaturation of fatty acids and blood phospholipids [44,45]. Oxysterol binding protein like 5 (OSBPL5) is a lipid transporter, chiefly linked with the exchange of phosphatidylserine with phosphatidylinositol 4-phosphate. It has a role in maintaining cholesterol balance [46,47].

These genes have not been previously identified by any GWAS as being related to fatty acid content and fat regulation. In the present GWAS, we did not identify any SNP windows which contain some genes (LXR, LXRA, SREBP1, PPARG, ACSL1, LEP, ACACA, FABP4, and SL1TRK6) previously shown to be associated with FA composition and variation in beef cattle [27,48-53]. This may indicate that genetic control of fatty acid content varies greatly from breed to breed.

Table 4. $1 \mathrm{Mb}$ chromosome windows having candidate gene associated with fatty acid traits.

\begin{tabular}{|c|c|c|c|}
\hline Sr. & Region (BTA_Mb) & Gene & Traits \\
\hline 1 & $6 \_7$ & FABP2 & $\mathrm{C} 16: 1 / \mathrm{C} 16: 0$ \\
\hline 2 & 10_19 & BBS4 & $\begin{array}{l}\text { C14:0, C14:1, C16:1, MCFA, LCFA, } \\
\text { C16:1/C16:0 }\end{array}$ \\
\hline 3 & $16 \_4$ & $\begin{array}{l}\text { PFKFB2, IL10, RAB7B, } \\
\text { PM20D1 }\end{array}$ & $\begin{array}{l}\text { SFA, MUFA, MUFA/SFA, UFA/SFA, } \\
\text { C16:1,C18:1/C16:0,C18:0 }\end{array}$ \\
\hline 4 & $19 \_42$ & THRA & C17:0 \\
\hline 5 & $19 \_51$ & FASN & $\begin{array}{l}\text { C14:0, C14:1, C16:0, C16:0/C14:0, } \\
\text { C16:1, C16:1/C16:0, C18:0/C16:0, } \\
\text { C18:1 c9, LCFA, MCFA, MUFA, } \\
\text { MUFA/SFA, SFA, UFA/SFA, } \\
\text { C16:1,C18:1/C16:0,C18:0 }\end{array}$ \\
\hline 6 & $24 \_49$ & ACAA2 & C17:0, C17:1 \\
\hline 7 & $26 \_21$ & SCD & $\begin{array}{l}\text { C14:0, C14:1, C14:1/C14:0, } \\
\text { C16:0/C14:0, C16:1, C16:1/C16:0, } \\
\text { C18:0, C18:1 c12, MUFA, } \\
\text { MUFA/SFA, SFA, UFA/SFA }\end{array}$ \\
\hline 8 & 29_18 & THRSP & $\begin{array}{l}\text { C14:0, C14:1, C16:0, C16:0/C14:0, } \\
\text { C16:1, C16:1/C16:0, C18:0, } \\
\text { C18:0/C16:0, C18:1 C9, LCFA, MCFA }\end{array}$ \\
\hline 9 & $29 \_42$ & FADS2, FADS3 & C16:0/C14:0 \\
\hline 10 & $29 \_49$ & MOB2, INS, OSBPL5 & C20:5, n3 \\
\hline
\end{tabular}

\subsection{Correlation within Genomic Regions}

All of the identified regions of the genome had a genetic correlation with a fatty acid trait that ranged from 0.1 to 0.74 . Both the highest genetic correlation of 0.74 and the lowest genetic correlation of 0.1 were observed for C13:0 (6_95 and 6_65). These genomic windows lack candidate genes associated with fatty acid composition or fat regulation. Some genomic regions $\left(6 \_7,10 \_19,16 \_4,19 \_42,19 \_51,24 \_49,26 \_21,29 \_18,29 \_42\right.$, and 29 49) that had high correlations with fatty acid content do contain potential candidate genes (FABP2, BBS4, PFKFB2, THRA, FASN, ACAA2, SCD, THRSP, FADS2, FADS3, MOB2, OSBPL5). Three of the genomic regions (19_51,26_21,29_18) were previously reported by our group to have a high correlation with fatty acid content [18]. These genomic windows harbor good candidate genes (FASN, SCD , THRSP) that may be associated with fatty acid composition $[27,28,49,54,55]$. Besides these three genomic windows, there are other genomic regions $\left(6 \_7,10 \_19,16 \_4,19 \_42,24 \_49,29 \_42\right.$, and 29_49) that had a positive correlation with different saturated or monounsaturated fatty acid traits (C14:0, C14:1, C16:1, C17:0, MCFA, LCFA) and fatty acid groups (C16:0/C14:0 and C16:1/C16:0). These regions have not been previously reported to be associated with fatty acid content, but they contain 
possible candidate genes ( $F A B P 2, B B S 4, T H R A, A C A A 2, F A D S 2$, and FADS3) for fatty acid synthesis/composition and fat regulation. [39,40,44,45,56-64].

\section{Conclusions}

Genome-wide association studies can provide insight into understanding the mechanisms underlying fatty acid composition. Furthermore, genomic selection methodology can be used to select for, and to alter, fatty acid content. This study utilized imputed BovineHD BeadChip (770k) genotypes along with skeletal muscle fatty acid content phenotypic data to identify $361-\mathrm{Mb}$ SNP windows that had a PPI $>0.90$. Some of these SNP windows have been previously reported, including 19_51,26_21, and 29_18. In addition, some new genomic regions that had not been previously reported to be associated with fatty acid content were identified: 6_7, 19_42, and 29_42. Fatty acid composition and deposition are complex polygenic traits having low to moderate heritability. The genomic regions identified in the present study and associated potential candidate genes for FA composition could help increase understanding of the genetic basis of FA composition in beef cattle (Black Angus). This study could also help to devise sensible breeding plans and selection strategies based on identified genomic regions for the improvement of beef fatty acid profile.

Author Contributions: Data curation, M.D.; Formal analysis, M.D. and L.M.K.; Funding acquisition, J.M.R.; Investigation, M.D.; Project administration, J.M.R.; Software, M.D. and L.M.K.; Supervision, J.M.R. and M.I.S.; Writing—original draft, M.D.; Writing—review \& editing, J.M.R. All authors have read and agreed to the published version of the manuscript.

Funding: This research was funded by Higher Education Commission of Pakistan under "International Research Support Initiative Program".

Institutional Review Board Statement: All the animal work and experiments were approved by the Iowa State University Animal Care and Use Committee (IACUC).

Data Availability Statement: Data will be available upon request.

Conflicts of Interest: The authors declare no conflict of interest.

\section{References}

1. Laborde, F.L.; Mandell, I.B.; Tosh, J.J.; Wilton, J.W.; Buchanan-Smith, J.G. Breed effects on growth performance, carcass characteristics, fatty acid composition, and palatability attributes in finishing steers. J. Anim. Sci. 2001, 79, 355-365. [CrossRef] [PubMed]

2. Vernooij, R.W.M.; Zeraatkar, D.; Han, M.A.; El Dib, R.; Zworth, M.; Milio, K.; Sit, D.; Lee, Y.; Gomaa, H.; Valli, C.; et al. Patterns of red and processed meat consumption and risk for cardiometabolic and cancer outcomes a systematic review and meta-analysis of cohort studies. Ann. Intern. Med. 2019, 171, 732-741. [CrossRef] [PubMed]

3. Valli, C.; Rabassa, M.; Johnston, B.C.; Kuijpers, R.; Prokop-Dorner, A.; Zajac, J.; Storman, D.; Storman, M.; Bala, M.M.; Solà, I.; et al. Health-related values and preferences regarding meat consumption a mixed-methods systematic review. Ann. Intern. Med. 2019, 171, 742-755. [CrossRef] [PubMed]

4. Han, M.A.; Zeraatkar, D.; Guyatt, G.H.; Vernooij, R.W.M.; El Dib, R.; Zhang, Y.; Algarni, A.; Leung, G.; Storman, D.; Valli, C.; et al. Reduction of red and processed meat intake and cancer mortality and incidence a systematic review and meta-analysis of cohort studies. Ann. Intern. Med. 2019, 171, 711-720. [CrossRef] [PubMed]

5. Zeraatkar, D.; Han, M.A.; Guyatt, G.H.; Vernooij, R.W.M.; El Dib, R.; Cheung, K.; Milio, K.; Zworth, M.; Bartoszko, J.J; Valli, C.; et al. Red and processed meat consumption and risk for all-cause mortality and cardiometabolic outcomes a systematic review and meta-analysis of cohort studies. Ann. Intern. Med. 2019, 171, 703-710. [CrossRef]

6. Zeraatkar, D.; Johnston, B.C.; Bartoszko, J.; Cheung, K.; Bala, M.M.; Valli, C.; Rabassa, M.; Sit, D.; Milio, K.; Sadeghirad, B.; et al. Effect of lower versus higher red meat intake on cardiometabolic and cancer outcomes a systematic review of randomized trials. Ann. Intern. Med. 2019, 171, 721-731. [CrossRef] [PubMed]

7. Johnston, B.C.; Zeraatkar, D.; Han, M.A.; Vernooij, R.W.M.; Valli, C.; El Dib, R.; Marshall, C.; Stover, P.J.; Fairweather-Taitt, S.; Wójcik, G.; et al. Unprocessed red meat and processed meat consumption: Dietary guideline recommendations from the nutritional recommendations (NUTRIRECS) consortium. Ann. Intern. Med. 2019, 171, 756-764. [CrossRef] [PubMed] 
8. Da Martins, T.S.; de Lemos, M.V.A.; Mueller, L.F.; Baldi, F.; de Amorim, T.R.; Ferrinho, A.M.; Muñoz, J.A.; de Fuzikawa, I.H.S.; de Moura, G.V.; Gemelli, J.L.; et al. Fat Deposition, Fatty Acid Composition, and Its Relationship with Meat Quality and Human Health. In Meat Science and Nutrition; IntechOpen: London, UK, 2018; pp. 17-37. Available online: https: //www.intechopen.com/chapters/61577 (accessed on 15 August 2021). [CrossRef]

9. Yen, C.-L.E.; Stone, S.J.; Koliwad, S.; Harris, C.; Farese, R.V. Thematic Review Series: Glycerolipids. DGAT enzymes and triacylglycerol biosynthesis. J. Lipid Res. 2008, 49, 2283-2301. [CrossRef]

10. Venkata Reddy, B.; Sivakumar, A.S.; Jeong, D.W.; Woo, Y.-B.; Park, S.-J.; Lee, S.-Y.; Byun, J.-Y.; Kim, C.-H.; Cho, S.-H.; Hwang, I. Beef quality traits of heifer in comparison with steer, bull and cow at various feeding environments. Anim. Sci. J. 2015, 86, 1-16. [CrossRef]

11. Vahmani, P.; Mapiye, C.; Prieto, N.; Rolland, D.C.; McAllister, T.A.; Aalhus, J.L.; Dugan, M.E.R. The scope for manipulating the polyunsaturated fatty acid content of beef: A review. J. Anim. Sci. Biotechnol. 2015, 6, 29. [CrossRef] [PubMed]

12. Cesar, A.S.M.; Regitano, L.C.A.; Mourão, G.B.; Tullio, R.R.; Lanna, D.P.D.; Nassu, R.T.; Mudado, M.A.; Oliveira, P.S.N.; do Nascimento, M.L.; Chaves, A.S.; et al. Genome-wide association study for intramuscular fat deposition and composition in Nellore cattle. BMC Genet. 2014, 15, 39. [CrossRef] [PubMed]

13. Kelly, M.J.; Tume, R.K.; Fortes, M.; Thompson, J.M. Whole-genome association study of fatty acid composition in a diverse range of beef cattle breeds. J. Anim. Sci. 2014, 92, 1895-1901. [CrossRef]

14. Wang, Z.; Zhu, B.; Niu, H.; Zhang, W.; Xu, L.; Xu, L.; Chen, Y.; Zhang, L.; Gao, X.; Gao, H.; et al. Genome wide association study identifies SNPs associated with fatty acid composition in Chinese Wagyu cattle. J. Anim. Sci. Biotechnol. 2019, 10, 27. [CrossRef]

15. Garmyn, A.J.; Hilton, G.G.; Mateescu, R.G.; Morgan, J.B.; Reecy, J.M.; Tait, J.G.; Beitz, D.C.; Duan, Q.; Schoonmaker, J.P.; Mayes, M.S.; et al. Estimation of relationships between mineral concentration and fatty acid composition of longissimus muscle and beef palatability traits. J. Anim. Sci. 2011, 89, 2849-2858. [CrossRef] [PubMed]

16. Sargolzaei, M.; Chesnais, J.P.; Schenkel, F.S. A new approach for efficient genotype imputation using information from relatives. BMC Genom. 2014, 15, 478. [CrossRef] [PubMed]

17. Kramer, L.M.; Abdel Ghaffar, M.A.; Koltes, J.E.; Fritz-Waters, E.R.; Mayes, M.S.; Sewell, A.D.; Weeks, N.T.; Garrick, D.J.; Fernando, R.L.; Ma, L.; et al. Epistatic interactions associated with fatty acid concentrations of beef from angus sired beef cattle. BMC Genom. 2016, 17, 891. [CrossRef] [PubMed]

18. Saatchi, M.; Garrick, D.J.; Tait, R.G.; Mayes, M.S.; Drewnoski, M.; Schoonmaker, J.; Diaz, C.; Beitz, D.C.; Reecy, J.M. Genome-wide association and prediction of direct genomic breeding values for composition of fatty acids in Angus beef cattlea. BMC Genom. 2013, 14, 730. [CrossRef] [PubMed]

19. Meuwissen, T.H.E.; Hayes, B.J.; Goddard, M.E. Prediction of total genetic value using genome-wide dense marker maps. Genetics 2001, 157, 1819-1829. [CrossRef] [PubMed]

20. Garrick, D.J.; Fernando, R.L. Implementing a QTL detection study (GWAS) using genomic prediction methodology. Methods Mol. Biol. 2013, 1019, 275-298. [CrossRef]

21. Habier, D.; Fernando, R.L.; Kizilkaya, K.; Garrick, D.J. Extension of the bayesian alphabet for genomic selection. BMC Bioinform. 2011, 12, 186. [CrossRef]

22. Kizilkaya, K.; Fernando, R.L.; Garrick, D.J. Genomic prediction of simulated multibreed and purebred performance using observed fifty thousand single nucleotide polymorphism genotypes. J. Anim. Sci. 2010, 88, 544-551. [CrossRef] [PubMed]

23. Chen, L.; Ekine-Dzivenu, C.; Vinsky, M.; Basarab, J.; Aalhus, J.; Dugan, M.E.R.; Fitzsimmons, C.; Stothard, P.; Li, C. Genome-wide association and genomic prediction of breeding values for fatty acid composition in subcutaneous adipose and longissimus lumborum muscle of beef cattle. BMC Genet. 2015, 16, 135. [CrossRef] [PubMed]

24. Das, P.J.; Chowdhary, B.P.; Raudsepp, T. Characterization of the bovine pseudoautosomal region and comparison with sheep, goat, and other mammalian pseudoautosomal regions. Cytogenet. Genome Res. 2009, 126, 139-147. [CrossRef] [PubMed]

25. Gellatly, S.A.; Kalujnaia, S.; Cramb, G. Cloning, tissue distribution and sub-cellular localisation of phospholipase C X-domain containing protein (PLCXD) isoforms. Biochem. Biophys. Res. Commun. 2012, 424, 651-656. [CrossRef]

26. Buchanan, J.W.; Reecy, J.M.; Garrick, D.J.; Duan, Q.; Beitz, D.C.; Koltes, J.E.; Saatchi, M.; Koesterke, L.; Mateescu, R.G. Deriving Gene Networks from SNP Associated with Triacylglycerol and Phospholipid Fatty Acid Fractions from Ribeyes of Angus Cattle. Front. Genet. 2016, 7, 116. [CrossRef]

27. Zhang, S.; Knight, T.J.; Reecy, J.M.; Beitz, D.C. DNA polymorphisms in bovine fatty acid synthase are associated with beef fatty acid composition. Anim. Genet. 2008, 39, 62-70. [CrossRef] [PubMed]

28. Narukami, T.; Sasazaki, S.; Oyama, K.; Nogi, T.; Taniguchi, M.; Mannen, H. Effect of DNA polymorphisms related to fatty acid composition in adipose tissue of Holstein cattle. Anim. Sci. J. 2011, 82, 406-411. [CrossRef]

29. Yokota, S.; Sugita, H.; Ardiyanti, A.; Shoji, N.; Nakajima, H.; Hosono, M.; Otomo, Y.; Suda, Y.; Katoh, K.; Suzuki, K. Contributions of FASN and SCD gene polymorphisms on fatty acid composition in muscle from Japanese Black cattle. Anim. Genet. 2012, 43, 790-792. [CrossRef]

30. Maharani, D.; Jung, Y.; Jung, W.Y.; Jo, C.; Ryoo, S.H.; Lee, S.H.; Yeon, S.H.; Lee, J.H. Association of five candidate genes with fatty acid composition in Korean cattle. Mol. Biol. Rep. 2012, 39, 6113-6121. [CrossRef]

31. Li, C.; Sun, D.; Zhang, S.; Wang, S.; Wu, X.; Zhang, Q.; Liu, L.; Li, Y.; Qiao, L. Genome wide association study identifies 20 novel promising genes associated with milk fatty acid traits in Chinese Holstein. PLoS ONE 2014, 9, e96186. [CrossRef] [PubMed] 
32. Donnelly, C.; Olsen, A.M.; Lewis, L.D.; Eisenberg, B.L.; Eastman, A.; Kinlaw, W.B. Conjugated linoleic acid (CLA) inhibits expression of the spot 14 (THRSP) and fatty acid synthase genes and impairs the growth of human breast cancer and liposarcoma cells. Nutr. Cancer 2009, 61, 114-122. [CrossRef]

33. La, B.; Oh, D.; Lee, Y.; Shin, S.; Lee, C.; Chung, E.; Yeo, J. Association of bovine fatty acid composition with novel missense nucleotide polymorphism in the thyroid hormone-responsive (THRSP) gene. Anim. Genet. 2013, 44, 118. [CrossRef] [PubMed]

34. Graugnard, D.E.; Berger, L.L.; Faulkner, D.B.; Loor, J.J. High-starch diets induce precocious adipogenic gene network up-regulation in longissimus lumborum of early-weaned Angus cattle. Br. J. Nutr. 2010, 103, 953-963. [CrossRef] [PubMed]

35. McClure, M.C.; Morsci, N.S.; Schnabel, R.D.; Kim, J.W.; Yao, P.; Rolf, M.M.; McKay, S.D.; Gregg, S.J.; Chapple, R.H.; Northcutt, S.L.; et al. A genome scan for quantitative trait loci influencing carcass, post-natal growth and reproductive traits in commercial Angus cattle. Anim. Genet. 2010, 41, 597-607. [CrossRef] [PubMed]

36. Long, J.Z.; Roche, A.M.; Berdan, C.A.; Louie, S.M.; Roberts, A.J.; Svensson, K.J.; Dou, F.Y.; Bateman, L.A.; Mina, A.I.; Deng, Z.; et al. Ablation of PM20D1 reveals N-acyl amino acid control of metabolism and nociception. Proc. Natl. Acad. Sci. USA 2018, 115, E6937-E6945. [CrossRef]

37. Long, J.Z.; Svensson, K.J.; Bateman, L.A.; Lin, H.; Kamenecka, T.; Lokurkar, I.A.; Lou, J.; Rao, R.R.; Chang, M.R.R.; Jedrychowski, M.P.; et al. The Secreted Enzyme PM20D1 Regulates Lipidated Amino Acid Uncouplers of Mitochondria. Cell 2016, 166, 424-435. [CrossRef] [PubMed]

38. Gunawardhana, L.P.; Baines, K.J.; Mattes, J.; Murphy, V.E.; Simpson, J.L.; Gibson, P.G. Differential DNA methylation profiles of infants exposed to maternal asthma during pregnancy. Pediatr. Pulmonol. 2014, 49, 852-862. [CrossRef] [PubMed]

39. Hu, G.; Wang, S.; Tian, J.; Chu, L.; Li, H. Epistatic effect between ACACA and FABP2 gene on abdominal fat traits in broilers. J. Genet. Genom. 2010, 37, 505-512. [CrossRef]

40. Estellé, J.; Mercadé, A.; Pérez-Enciso, M.; Pena, R.N.; Silió, L.; Sánchez, A.; Folch, J.M. Evaluation of FABP2 as candidate gene for a fatty acid composition QTL in porcine chromosome 8. J. Anim. Breed. Genet. 2009, 126, 52-58. [CrossRef] [PubMed]

41. Aksanov, O.; Green, P.; Birk, R.Z. BBS4 directly affects proliferation and differentiation of adipocytes. Cell. Mol. Life Sci. 2014, 71, 3381-3392. [CrossRef] [PubMed]

42. Prieto-Echagüe, V.; Lodh, S.; Colman, L.; Bobba, N.; Santos, L.; Katsanis, N.; Escande, C.; Zaghloul, N.A.; Badano, J.L. BBS4 regulates the expression and secretion of FSTL1, a protein that participates in ciliogenesis and the differentiation of 3T3-L1. Sci. Rep. 2017, 7, 9765. [CrossRef] [PubMed]

43. Miltiadou, D.; Hager-Theodorides, A.L.; Symeou, S.; Constantinou, C.; Psifidi, A.; Banos, G.; Tzamaloukas, O. Variants in the $3^{\prime}$ untranslated region of the ovine acetyl-coenzyme A acyltransferase 2 gene are associated with dairy traits and exhibit differential allelic expression. J. Dairy Sci. 2017, 100, 6285-6297. [CrossRef] [PubMed]

44. Rzehak, P.; Heinrich, J.; Klopp, N.; Schaeffer, L.; Hoff, S.; Wolfram, G.; Illig, T.; Linseisen, J. Evidence for an association between genetic variants of the fatty acid desaturase 1 fatty acid desaturase 2 (FADS1 FADS2) gene cluster and the fatty acid composition of erythrocyte membranes. Br. J. Nutr. 2009, 101, 20-26. [CrossRef]

45. Park, W.J.; Kothapalli, K.S.D.; Reardon, H.T.; Kim, L.Y.; Brenna, J.T. Novel fatty acid desaturase 3 (FADS3) transcripts generated by alternative splicing. Gene 2009, 446, 28-34. [CrossRef] [PubMed]

46. Chung, J.; Torta, F.; Masai, K.; Lucast, L.; Czapla, H.; Tanner, L.B.; Narayanaswamy, P.; Wenk, M.R.; Nakatsu, F.; De Camilli, P. PI4P/phosphatidylserine countertransport at ORP5- and ORP8-mediated ER-Plasma membrane contacts. Science 2015, 349, 428-432. [CrossRef] [PubMed]

47. Pollock, R.A.; Zaman, L.; Chandran, V.; Gladman, D.D. Epigenome-wide analysis of sperm cells identifies IL22 as a possible germ line risk locus for psoriatic arthritis. PLoS ONE 2019, 14, e0212043. [CrossRef] [PubMed]

48. Shi, H.; Kokoeva, M.V.; Inouye, K.; Tzameli, I.; Yin, H.; Flier, J.S. TLR4 links innate immunity and fatty acid-induced insulin resistance. J. Clin. Investig. 2006, 116, 3015-3025. [CrossRef] [PubMed]

49. Matsuhashi, T.; Maruyama, S.; Uemoto, Y.; Kobayashi, N.; Mannen, H.; Abe, T.; Sakaguchi, S.; Kobayashi, E. Effects of bovine fatty acid synthase, stearoyl-coenzyme A desaturase, sterol regulatory element-binding protein 1, and growth hormone gene polymorphisms on fatty acid composition and carcass traits in Japanese Black cattle. J. Anim. Sci. 2011, 89, 12-22. [CrossRef] [PubMed]

50. Oh, D.; Lee, Y.; Lee, C.; Chung, E.; Yeo, J. Association of bovine fatty acid composition with missense nucleotide polymorphism in exon7 of peroxisome proliferator-activated receptor gamma gene. Anim. Genet. 2012, 43, 474. [CrossRef]

51. Widmann, P.; Nuernberg, K.; Kuehn, C.; Weikard, R. Association of an ACSL1 gene variant with polyunsaturated fatty acids in bovine skeletal muscle. BMC Genet. 2011, 12, 96. [CrossRef] [PubMed]

52. Orrù, L.; Cifuni, G.F.; Piasentier, E.; Corazzin, M.; Bovolenta, S.; Moioli, B. Association analyses of single nucleotide polymorphisms in the LEP and SCD1 genes on the fatty acid profile of muscle fat in Simmental bulls. Meat Sci. 2011, 87, 344-348. [CrossRef] [PubMed]

53. Hoashi, S.; Hinenoya, T.; Tanaka, A.; Ohsaki, H.; Sasazaki, S.; Taniguchi, M.; Oyama, K.; Mukai, F.; Mannen, H. Association between fatty acid compositions and genotypes of FABP4 and LXR-alpha in Japanese Black cattle. BMC Genet. 2008, 9, 84. [CrossRef]

54. Abe, T.; Saburi, J.; Hasebe, H.; Nakagawa, T.; Misumi, S.; Nade, T.; Nakajima, H.; Shoji, N.; Kobayashi, M.; Kobayashi, E. Novel mutations of the FASN gene and their effect on fatty acid composition in japanese black beef. Biochem. Genet. 2009, 47, 397-411. [CrossRef] [PubMed] 
55. Uemoto, Y.; Abe, T.; Tameoka, N.; Hasebe, H.; Inoue, K.; Nakajima, H.; Shoji, N.; Kobayashi, M.; Kobayashi, E. Whole-genome association study for fatty acid composition of oleic acid in Japanese Black cattle. Anim. Genet. 2011, 42, 141-148. [CrossRef] [PubMed]

56. Mullur, R.; Liu, Y.Y.; Brent, G.A. Thyroid hormone regulation of metabolism. Physiol. Rev. 2014, 94, 355-382. [CrossRef]

57. Yao, D.W.; Luo, J.; He, Q.Y.; Wu, M.; Shi, H.B.; Wang, H.; Wang, M.; Xu, H.F.; Loor, J.J. Thyroid hormone responsive (THRSP) promotes the synthesis of medium-chain fatty acids in goat mammary epithelial cells. J. Dairy Sci. 2016, 99, 3124-3133. [CrossRef]

58. Damiano, F.; Rochira, A.; Gnoni, A.; Siculella, L. Action of thyroid hormones, T3 and T2, on hepatic fatty acids: Differences in metabolic effects and molecular mechanisms. Int. J. Mol. Sci. 2017, 18, 744. [CrossRef]

59. Boschetti, E.; Bordoni, A.; Meluzzi, A.; Castellini, C.; Dal Bosco, A.; Sirri, F. Fatty acid composition of chicken breast meat is dependent on genotype-related variation of FADS1 and FADS2 gene expression and desaturating activity. Animal 2016, 10, 700-708. [CrossRef]

60. Schaeffer, L.; Gohlke, H.; Müller, M.; Heid, I.M.; Palmer, L.J.; Kompauer, I.; Demmelmair, H.; Illig, T.; Koletzko, B.; Heinrich, J. Common genetic variants of the FADS1 FADS2 gene cluster and their reconstructed haplotypes are associated with the fatty acid composition in phospholipids. Hum. Mol. Genet. 2006, 15, 1745-1756. [CrossRef]

61. Jornayvaz, F.R.; Lee, H.Y.; Jurczak, M.J.; Alves, T.C.; Guebre-Egziabher, F.; Guigni, B.A.; Zhang, D.; Samuel, V.T.; Silva, J.E.; Shulman, G.I. Thyroid hormone receptor- $\alpha$ gene knockout mice are protected from diet-induced hepatic insulin resistance. Endocrinology 2012, 153, 583-591. [CrossRef] [PubMed]

62. Yao, X.; Hou, S.; Zhang, D.; Xia, H.; Wang, Y.C.; Jiang, J.; Yin, H.; Ying, H. Regulation of fatty acid composition and lipid storage by thyroid hormone in mouse liver. Cell Biosci. 2014, 4, 38. [CrossRef] [PubMed]

63. Sinha, R.A.; Singh, B.K.; Yen, P.M. Thyroid hormone regulation of hepatic lipid and carbohydrate metabolism. Trends Endocrinol. Metab. 2014, 25, 538-545. [CrossRef] [PubMed]

64. Rudolph, M.C.; Monks, J.; Burns, V.; Phistry, M.; Marians, R.; Foote, M.R.; Bauman, D.E.; Anderson, S.M.; Neville, M.C. Sterol regulatory element binding protein and dietary lipid regulation of fatty acid synthesis in the mammary epithelium. Am. J. Physiol. Endocrinol. Metab. 2010, 299, 918-927. [CrossRef] [PubMed] 